\title{
Identification of differentially expressed genes in asthma by bioinformatics analysis
}

Wang Zhaojun ${ }^{1 \#}$, Mo Zhifeng ${ }^{2 \#}$, Liang Hongsen ${ }^{1}$, Zhang Qiwei ${ }^{1}$, Li Wei ${ }^{1}$, Yan Dongqing ${ }^{1}$, Yin Yin ${ }^{1}$, Fan Haiyang ${ }^{1}$, Zhang Liang ${ }^{1}$, Shi Donglei ${ }^{1}$, Zhang Junhang ${ }^{1 *}$, Li Haifeng ${ }^{3 *}$

${ }^{1}$ Department of thoracic surgery, The Seventh Affiliated Hospital, Sun Yat-sen University, Shenzhen 518107, China ${ }^{2}$ Emergency Department, the Seventh Affiliated Hospital, Sun Yat-sen University, Shen Zhen 518107, China

${ }^{3}$ Department of Anesthesiology, Guangdong General Hospital, Guangzhou 510080, China

Zhang Jun-hang, E-mail: zhangjh33@mail.sysu.edu.cn, https://orcid.org/0000-0002-7979-6093

Li Hai-feng, E-mail: lihaifeng2016@yeah.net, https://orcid.org/0000-0001-9111-4377

${ }^{*}$ Wang Zhaojun and Zhifeng Mo contributed to this study equally.

*Zhang Junhang and Li Haifeng are the corresponding author.

Article History Received 18 April 2021 Accepted 25 May 2021 Published 30 June 2021

Cite this Article Wang Zhaojun, Mo Zhifeng, Liang Hongsen, Zhang Qiwei, Li Wei, Yan Dongqing, Yin Yin, Fan Haiyang, Zhang Liang, Shi Donglei, Zhang Junhang, Li Haifeng. Identification of differentially expressed genes in asthma by bioinformatics analysis [J].Medical Research, 2021.3(2):28-36, http://dx.doi.org/10.6913/MRHK.202106_3

(2). 0004

Copyright (C) 2021 Creative Publishing Co., Limited. All rights reserved. Email:mrhk26640333@gmail.com.

\section{ABSTRACT}

Objective Asthma is a common inflammatory disease of the airway, and its underlying mechanism is complex. The role of microRNAs (miRNAs) in asthma is unclear. The present study aimed to investigate miRNA-mRNA regulatory networks underlying asthma.

Methods One microarray dataset was downloaded from the Gene Expression Omnibus (GEO) database. Differential expression of miRNAs (DEMs) was identified in bronchial epithelial cells (BECs) isolated from healthy donors and patients with asthma. MiRTarBase, mirDIP, and miRDB were used to predict target genes, followed by protein-protein interaction (PPI) network analysis, Kyoto Encyclopedia of Genes and Genomes (KEGG) analysis, and Gene Ontology (GO) analysis; cytoHubba was used to predict the important nodes in the network. The miRNA-hub genes sub-network of interest was determined.

Results This study constructed an asthma-associated miRNA-mRNA network, in which seven key miRNAs and 10 hub genes were identified.

Conclusions The novel miRNAs and hub genes identified in the present study could be potential diagnostic and treatment biomarkers for asthma.

Keywords GEO; miRNA; asthma; bioinformatics

\section{INTRODUCTION}

Asthma is a chronic disorder of the airway, and airway inflammation plays a critical role in asthma pathogenesis. Better understanding of the pathogenesis of asthma has enabled great progress in treating patients with asthma. It has been estimated that 339 million people worldwide are affected by asthma annually, resulting in huge spending on health services ${ }^{[1]}$. The various roles of microRNAs (miRNAs) in the regulation of human diseases are receiving increasing attention, and a better understanding of these roles 28

Copyright (C) 2021 Creative Publishing Co., Limited. All rights reserved. Email:mrhk26640333@gmail.com 
may enable to identify new targets for asthma treatment ${ }^{[2]}$.

miRNAs are a class of small nonprotein-coding RNAs that are 20-30 nucleotides in length ${ }^{[3]}$. In recent years, miRNAs have received wide attention, as they are involved in many biological processes, including tissue and organ development, biochemical pathways, cell signaling, and several other mechanisms ${ }^{[4]}$. There are a large number of miRNAs, some of which are considered to be useful diagnostic and prognostic tools in cancer and other diseases ${ }^{[3]}$. miRNAs are present in airway biopsy cells, epithelial brushing cells, sera/plasma, sputum, bronchoalveolar lavage (BAL), and exosomes ${ }^{[5]}$. Deregulation of some miRNAs is thought to play a role in the pathogenesis of asthma. Jimenez et al found that miRNAs are expressed differently in human monocyte-derived macrophages (MDMs) under different conditions; miRNA-155, miRNA-339, and miRNA-200a were downregulated, while miRNA-21, miRNA-146b-5p, miRNA-27a, and miRNA-22 were upregulated in M2c phenotype. miRNA-181a/b and miRNA-147b were downregulated, while miRNA-500a, miRNA-511, and miRNA-502 were upregulated in M2a phenotype ${ }^{[6]}$. Compared to the control group, miRNA-18A was downregulated in nasal mucosal biopsies of patients with asthma ${ }^{[7]}$. An in vitro study showed that miRNA-20a-5p levels were downregulated in the bronchial epithelial brushing cells in patients with severe asthma compared to cells from healthy donors ${ }^{[8]}$.

In the present study, we aimed to identify differential expression of miRNAs (DEMs) by using the Gene Expression Omnibus (GEO) database, predicted mRNAs were recorded, followed by protein-protein interaction (PPI) network analysis to detect potential genes. We performed Kyoto Encyclopedia of Genes and Genomes (KEGG) enrichment analysis and Gene Ontology (GO) annotation for further understanding of the mRNAs. Finally, an miRNA-mRNA network for asthma was successfully established.

\section{MATERIALS AND METHODS}

\section{Source of the microarray data}

The dataset GSE25230 was downloaded from the public GEO database, which is a functional genomic database. This dataset included seven healthy and seven asthmatic donors from whom bronchial epithelial cells (BECs) were isolated. The samples were tested by the GPL8786 [miRNA-1] Affymetrix Multispecies miRNA-1 Array.

\section{Identification of DEMs}

Differential analyses of GSE25230 was performed to identify DEMs between healthy and asthmatic donors using the online tool GEO2R ${ }^{[9]}$. The criterion for DEMs was $\mathrm{P}<0.05$ and $|\log F C| \geq 3$. A volcano plot of the DECs was constructed using the Sangerbox.

\section{Identification of target genes and construction of the miRNA-mRNA network}

The target genes were predicted using the following three databases: mirTarBase (http://mirtarbase.cuhk.edu.cn), mirDIP (http://ophid.utoronto.ca/mirDIP/index.jsp), and miRDB (http://mirdb.org/index.html). The target genes were screened according to the overlapping results of the three databases. An miRNA-mRNA network was constructed. The network of miRNA-mRNA interactions was visualized by Cytoscape. 


\section{KEGG pathway enrichment and GO analyses}

KEGG pathway enrichment analysis and GO annotation of the target genes were performed. GO annotation included three aspects: biological process (BP), cellular component (CC), and molecular function (MF) ${ }^{[10]}$. Annotation, visualization, and integrated discovery were based on the online DAVID database (http://mirdb.org/index.html). P value of $<0.05$ was considered to be significant.

\section{PPI network and validation of the hub genes}

The STRING database (https://string-db.org) was used for the PPI network. The top 10 genes were considered as hub genes in Cytoscape software by the CytoHubba app. The miRNA-hub genes sub-network of interest was determined.

\section{RESULT}

\section{Identification of potential DEMs}

To find the potential miRNAs underlying asthma development, the miRNA dataset GSE25230 was selected. The GEO2R tool was used to perform differential expression analysis provided by the GEO Database. As shown in Table 1, eight upregulated DEMs were screened out on the basis of the selection criterion of $|\log 2 \mathrm{FC}| \geq 3$ and $\mathrm{P}<0.05$. The volcano plot of DEMs is shown in Figure 1 .

Table 1. The DEMs screen from GEO datasets

\begin{tabular}{ccccc}
\hline miRNA_ID & adj.P.Val & $\mathrm{t}$ & $\mathrm{B}$ & $\log \mathrm{FC}$ \\
\hline hsa-mir-487b & 0.00958 & 5.47828 & 2.43 & 3.318917 \\
hsa-mir-129-2 // hsa-mir-129-1 & 0.00958 & 5.365634 & 2.211 & 3.411713 \\
hsa-mir-374a & 0.00958 & 5.199347 & 1.884 & 3.135287 \\
hsa-mir-335 & 0.00958 & 5.161434 & 1.809 & 3.492887 \\
hsa-mir-181a-1 // hsa-mir-181a-2 & 0.00958 & 5.119879 & 1.726 & 3.355209 \\
hsa-mir-576 & 0.00958 & 5.119417 & 1.725 & 3.528878 \\
\hline
\end{tabular}

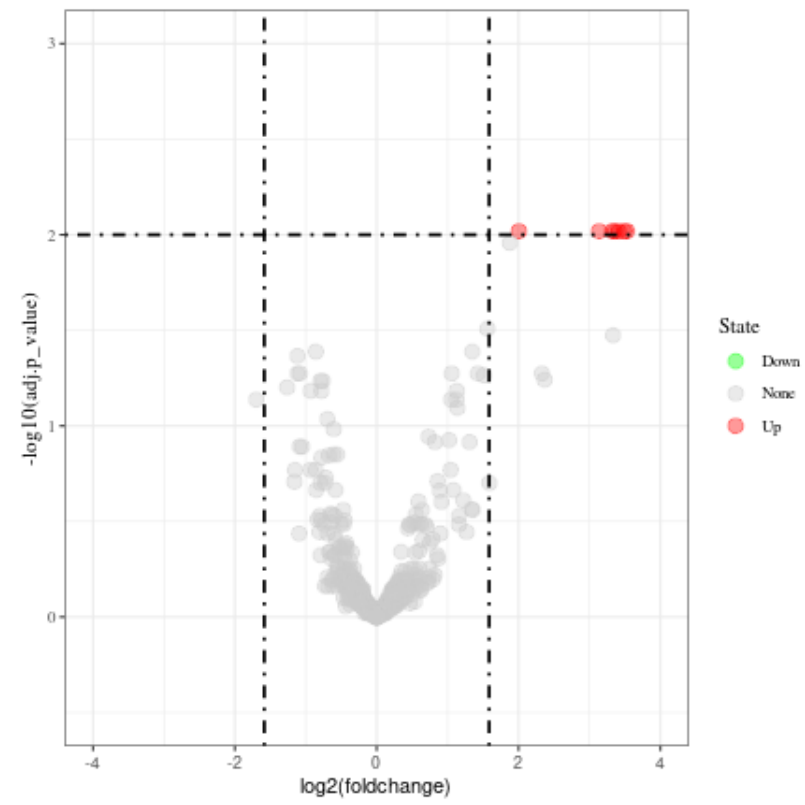

Figure 1. Volcano plot of differentially expressed miRNAs in GSE25230. 


\section{Identification of mRNAs and construction of the miRNA-mRNA network}

Target genes that might bind to the DEMs were predicted using the miRTarBase, mirDIP, and miRDB databases. Because there were no target genes of hsa-miR-374a-3p, the DEM hsa-miR-374a-3p was excluded, and the remaining seven DEMs were used to predict target genes (Table 2). An overlap of 391 target genes from seven DEMs was observed among the three databases. The network of miRNA-mRNA interactions was visualized by Cytoscape (Figure 2).

Table 2. Seven DEMs used to predict target genes

\begin{tabular}{cc}
\hline number & miRNAs \\
\hline 1 & hsa-miR-129-1-3p \\
2 & hsa-miR-129-2-3p \\
3 & hsa-miR-181a-5p \\
4 & hsa-miR-335-5p \\
5 & hsa-miR-374a-5p \\
6 & hsa-miR-487b-3p \\
7 & hsa-miR-576-5p \\
\hline
\end{tabular}

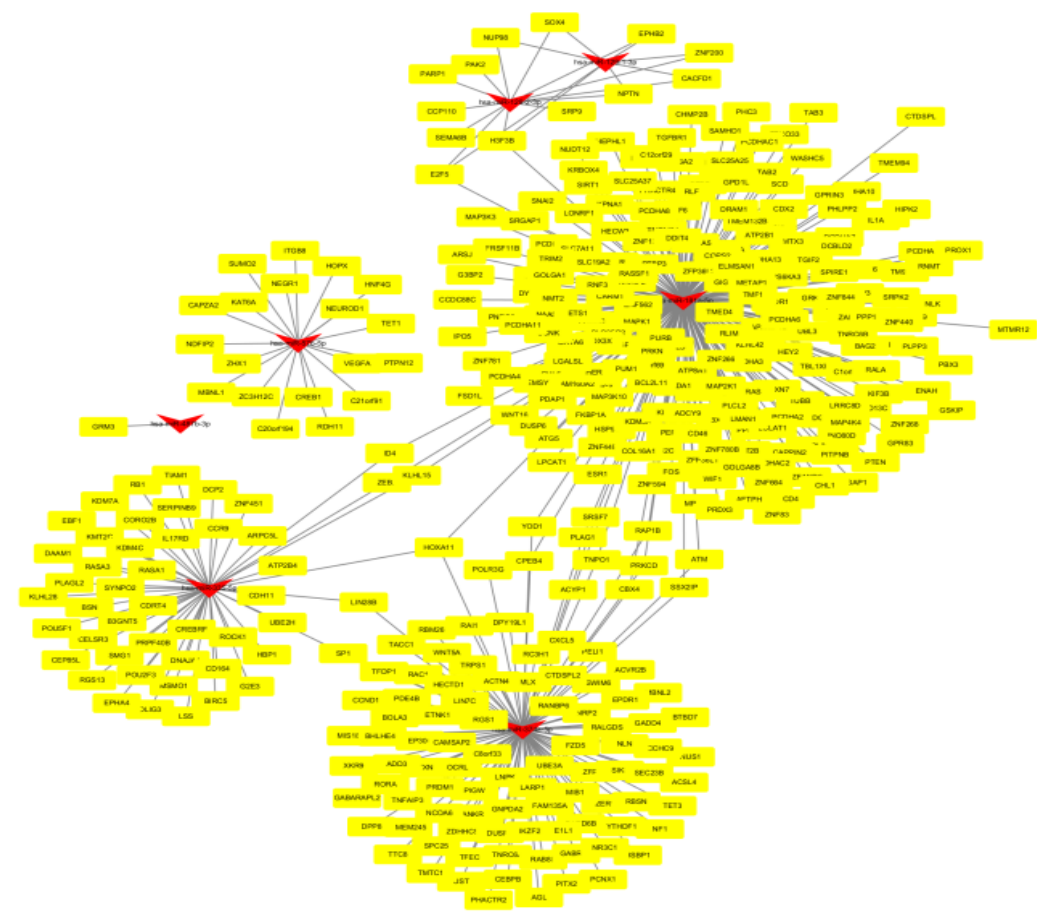

Figure 2. Interaction networks of miRNA-mRNA. The red dot represents miRNAs and the yellow dot represents target genes.

\section{KEGG pathway enrichment and GO analyses}

GO annotation and KEGG pathway enrichment analyses were performed for the target genes (Figure 3). In the MF category, the main enriched category was "transcription factor binding." In the BP category, the main enriched category was "positive regulation of transcription from RNA polymerase II promoter." In the $\mathrm{CC}$ category, the main enriched category was "nucleus"(Figure 3B). In the KEGG pathway analysis, the main enriched pathway was "MAPK signaling pathway" (Figure 3A). 


\section{KEGG enrichment}

hsa05215:Prostate cancer hsa04320:Dorso-ventral axis formation hsa04350:TGF-beta signaling pathway hsa05212:Pancreatic cancer hsa05200:Pathways in cancer hsa05210:Colorectal cancer hsa04010:MAPK signaling pathway

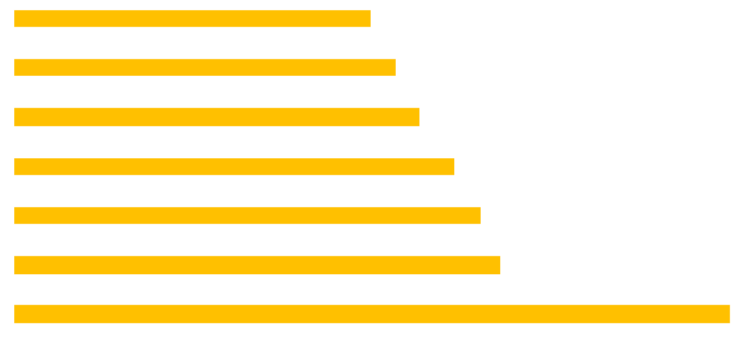

0

$\begin{array}{crrrrr}0.5 & 1 & 1.5 & 2 & 2.5 & 3 \\ -\log 10 \mathrm{FDR} & & & & & \end{array}$

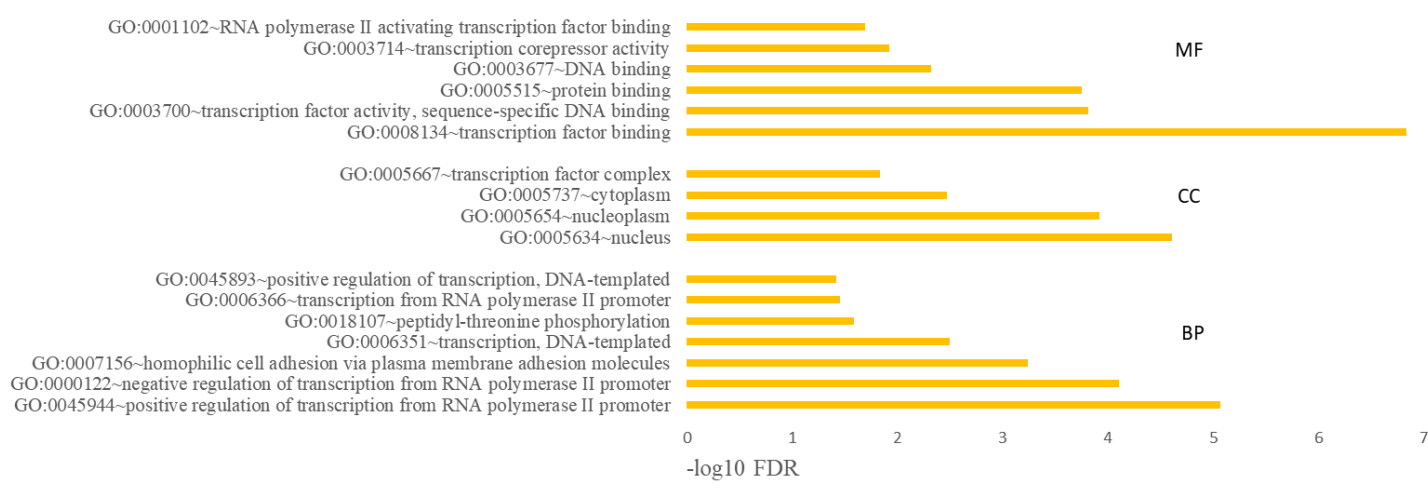

Figure 3. GO and KEGG analysis. BP: biological process; CC: cellular component; MF: molecular function.

\section{PPI network and validation of the hub genes}

The 391 target genes were used to construct the PPI network by using the STRING database (Figure 4). To identify the key module in the PPI Network, the top 10 hub genes were selected using CytoHubba based on the STRING database (Figure 5). Finally, the miRNA-hub genes sub-network of interest was determined (Figure 6).

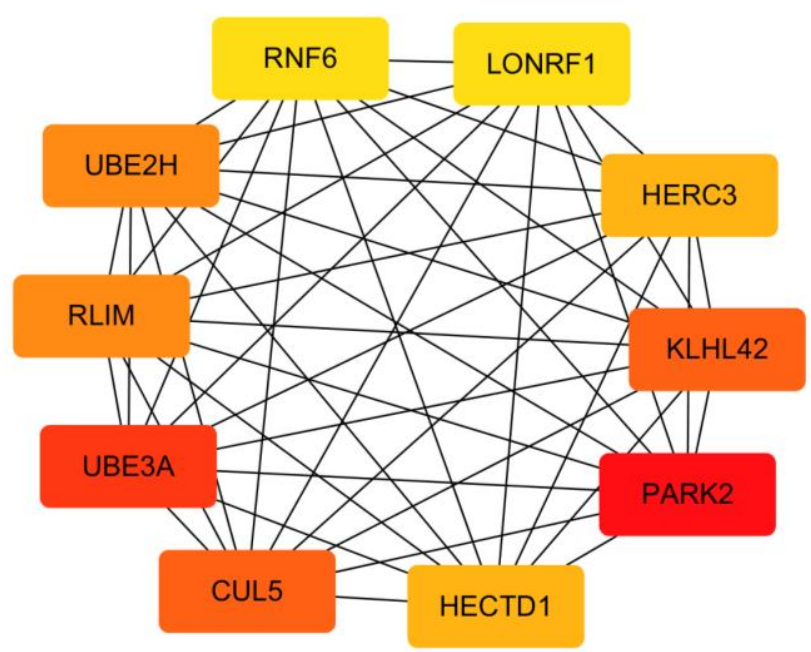

Figure 5. The interaction of 10 hub genes. 


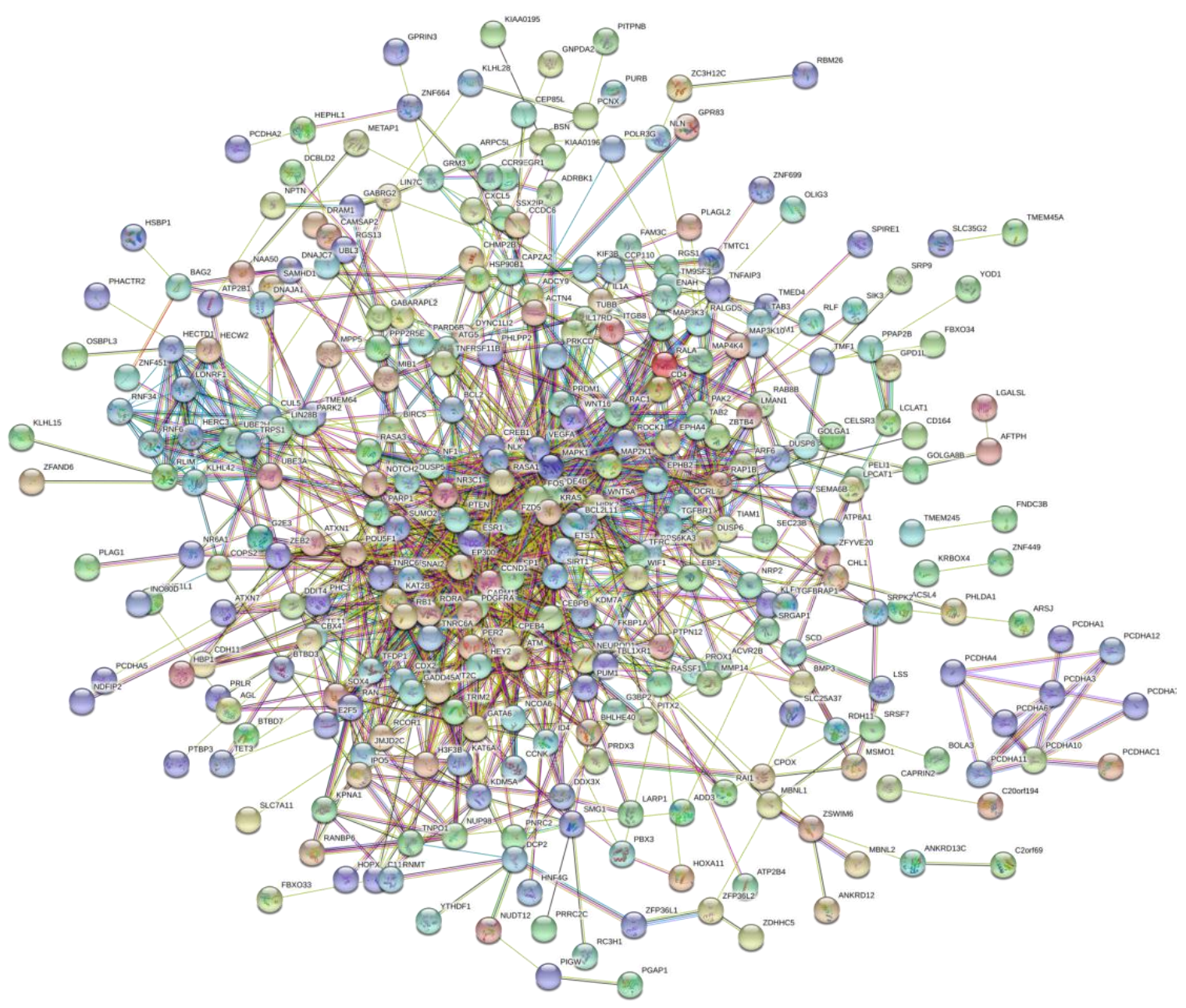

Figure 4. The PPI network.
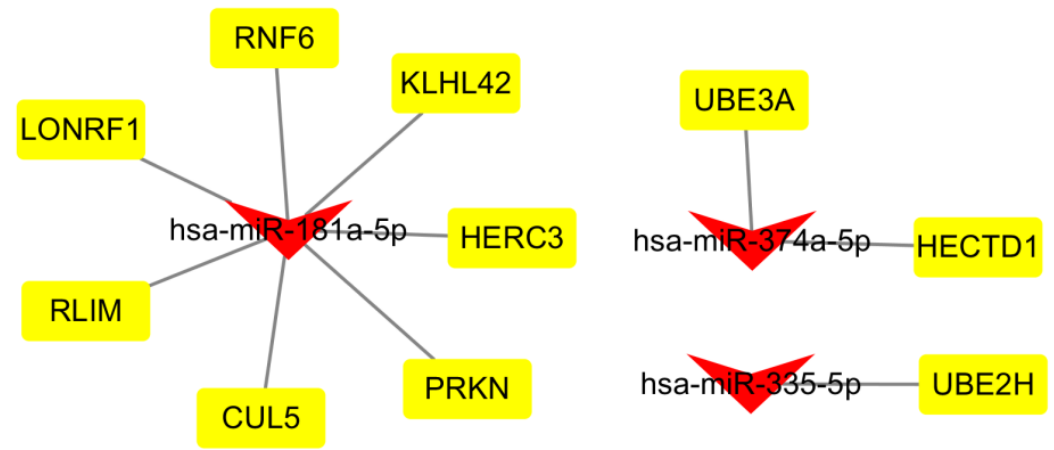

Figure 6. Interaction networks of miRNA-hub genes. The red dot represents miRNAs and the yellow dot represents target mRNAs.

\section{DISCUSSION}

Asthma is one of the most common chronic diseases and has become an economic burden worldwide ${ }^{[11]}$. In recent years, a better understanding of the pathogenesis of asthma has enabled great progress in the treatment of patients ${ }^{[12]}$. Asthma significantly reduces the respiratory function and activity of patients. The Copyright $\mathbb{C} 2021$ Creative Publishing Co., Limited.All rights reserved.Email:mrhk26640333@gmail.com 
diagnosis of asthma largely depends on clinical examination. Moreover, pathological tests do not appear to be useful in diagnosing asthma. Airway BECs are increasingly being considered as important components in the pathophysiology of asthma ${ }^{[5]}$. It has been demonstrated that providing molecular markers for early screening would be a convenient diagnostic method, and BECs could be a convenient source of biomarkers for asthma. The easy detection of miRNA makes it a suitable molecular marker for the early diagnosis and prognosis of various diseases.

miRNAs as a biomarker for the clinical diagnosis of lung diseases are receiving increasing attention [13-14]. The role of miRNA signaling pathways in asthma has been explained in detail ${ }^{[15]}$. The level of miR-744 was reduced in BECs from patients with severe asthma as compared to that in BECs from control subjects, and miR-744 downregulated the protein levels of TGF-B1 ${ }^{[16-18]}$. In an asthma mouse model, miR-943-3p was found to regulate the level of SFRP4 ${ }^{[19]}$. Several miRNAs are associated with the regulation of proliferation and function of smooth muscle cells, including miR-140 [20], miR-206 [21], miR-26 [22], miR-133a ${ }^{[23]}$, and miR-221 ${ }^{[24]}$. Chen et al reported that miR-620 increased TGF- $\beta 1$-mediated proliferation and inhibited apoptosis of airway smooth muscle cells by regulating the expression of PTEN and AKT ${ }^{[25]}$. A study reported that the expression of miR-19a was upregulated and the proliferation of BECs was enhanced by targeting TGF- $\beta$ receptor 2 , while the inhibition of miR-19a expression increased SMAD3 and decreased the proliferation of BECs ${ }^{[26]}$. The imbalance of miR-142-5P and miR-130a-3P is an important factor in regulating macrophage polarization and related asthma airway remodeling in ovalbumin-sensitized mice ${ }^{[27]}$.

In the present study, we identified DEMs according to the GEO data, confirmed seven miRNAs, predicted the mRNAs of miRNAs, and used the PPI network to identify the top 10 hub genes. We constructed an miRNA-mRNA network for asthma by using bioinformatics tools, and finally identified seven potential miRNAs and extracted 10 hub genes. Currently, there are few studies on miRNAs and hub genes in asthma, except for PARK2. PARK2 expression is upregulated in asthmatic airway epithelium and mediates mtDNA-induced airway inflammation ${ }^{[28]}$. However, further experimental confirmation is required.

In conclusion, based on bioinformatic analysis, we identified seven DEMs in asthma, and detected 10 hub genes that may serve as potential biomarkers of asthma. Our findings might provide biomarkers for the diagnosis and treatment of asthma.

\section{ACKNOWLEDGEMENT}

We thank International Science Editing (http://www.internationalscienceediting.com) for editing this manuscript.

\section{AUTHORS' CONTRIBUTIONS}

WZJ wrote the manuscript. ZJH conceived the study and provided guidance. All other authors contributed to the analysis and reviewed the manuscript.

\section{CONFLICTS OF INTEREST}

We declare no competing interests. 


\section{REFERENCES}

1 Global Asthma Network, The Global Asthma Report 2018, Auckland, New Zealand, 2018.

2 Maneechotesuwan K. Role of microRNA in severe asthma [J]. Respir Investig 2019; 57(1):9-19.

3 Feketea G, Bocsan CI, Popescu C, et al. A Review of Macrophage MicroRNAs' Role in Human Asthma [J]. Cells 2019; 8(5).

4 Taka S, Tzani-Tzanopoulou P, Wanstall H, et al. MicroRNAs in Asthma and Respiratory Infections: Identifying Common Pathways [J]. Allergy Asthma Immunol Res 2020; 12(1):4-23.

5 Jurj A, Pop L, Petrushev B, et al. Exosome-carried microRNA-based signature as a cellular trigger for the evolution of chronic lymphocytic leukemia into Richter syndrome [J]. Crit Rev Clin Lab Sci 2018; 55(7):501-515.

6 Cobos JV, Bradley EJ, Willemsen AM, et al. Next-generation sequencing of microRNAs uncovers expression signatures in polarized macrophages [J]. Physiol Genomics 2014; 46(3):91-103.

7 Suojalehto H, Lindstrom I, Majuri ML, et al. Altered microRNA expression of nasal mucosa in long-term asthma and allergic rhinitis [J]. Int Arch Allergy Immunol 2014; 163(3):168-78.

8 Martinez-Nunez RT, Rupani H, Plate M, et al. Genome-Wide Posttranscriptional Dysregulation by MicroRNAs in Human Asthma as Revealed by Frac-seq [J]. J Immunol 2018; 201(1):251-263.

9 Qin Y, Li L, Wang F, et al. Knockdown of Mir-135b Sensitizes Colorectal Cancer Cells to Oxaliplatin-Induced Apoptosis ThroughIncrease of FOXO1 [J]. Cell Physiol Biochem. 2018;48:1628-37.

10 Ashburner M, Ball CA, Blake JA, et al. Gene ontology: tool for the unification of biology. The Gene Ontology Consortium [J]. Nat Genet. 2000; 25:25-9.

$11 \mathrm{Du}$ J, Yuan Z, Ma Z et al. KEGG-PATH: Kyoto encyclopedia of genes and genomes-based pathway analysis using a path analysis model [J]. Mol Biosyst. 2014; 10:2441-7.

12 Loftus PA, Wise SK. Epidemiology and economic burden of asthma. Int Forum Allergy Rhinol [J]. 2015;5(Suppl 1):S7-10.

13 Holgate ST. The sentinel role of the airway epithelium in asthma pathogenesis [J]. Immunol Rev 2011; 242(1):205-19.

14 Fahy JV, Locksley RM. The airway epithelium as a regulator of Th2 responses in asthma [J]. Am J Respir Crit Care Med 2011; 184(4):390-2.

15 Boateng E, Krauss-Etschmann S. miRNAs in Lung Development and Diseases [J]. Int J Mol Sci 2020; 21(8).

16 Mousavi SR, Ahmadi A, Jamalkandi SA, Salimian J. Involvement of microRNAs in physiological and pathological processes in asthma [J]. J Cell Physiol 2019; 234(12):21547-21559.

17 Taka S, Tzani-Tzanopoulou P, Wanstall H, et al. MicroRNAs in Asthma and Respiratory Infections: Identifying Common Pathways [J]. Allergy Asthma Immunol Res 2020; 12(1):4-23.

18 Huang H, Lu H, Liang L, et al. MicroRNA-744 Inhibits Proliferation of Bronchial Epithelial Cells by Regulating Smad3 Pathway via Targeting Transforming Growth Factor-beta1 (TGF-beta1) in Severe Asthma Copyright $\odot 2021$ Creative Publishing Co., Limited.All rights reserved.Email:mrhk26640333@gmail.com 
[J]. Med Sci Monit 2019; 25:2159-2168.

19 Shen J, Zhao J, Ye Q Y, et al. Interference of miR-943-3p with secreted frizzled-related proteins4 (SFRP4) in an asthma mouse model[J]. Cell Tissue Res. 2019, 378(1): 67-80.

20 Jude JA, Dileepan M, Subramanian S, et al. miR-140-3p regulation of TNF- $\alpha$-inducedCD38 expression in human airway smooth muscle cells [J]. Am JPhysiol Lung Cell Mol Physiol. 2012;303:L460-8.

21 Radzikinas K, Aven L, Jiang Z, et al. A Shh/miR-206/BDNF cascade coordinates innervation andformation of airway smooth muscle [J]. J Neurosci. 2011;31:15407-15.

22 Mohamed JS, Lopez MA, Boriek AM. Mechanical stretch up-regulates microRNA-26a and induces human airway smooth mus-cle hypertrophy by suppressing glycogen synthase kinase-3 $\beta$ [J].JBiol Chem. 2010;285:29336-47.

23 Chiba Y, Tanabe M, Goto K, et al. Down-regulationof miR-133a contributes to up-regulation of Rhoa in bronchialsmooth muscle cells [J]. Am J Respir Crit Care Med. 2009;180:713-9.

24 Perry MM, Baker JE, Gibeon DS, et al. Airwaysmooth muscle hyperproliferation is regulated by microRNA-221in severe asthma [J]. Am J Respir Cell Mol Biol. 2014;50:7-1.

25 Chen H, Guo SX, Zhang S, et al. MiRNA-620 promotes TGF-beta1-induced proliferation of airway smooth muscle cell through controlling PTEN/AKT signaling pathway [J]. Kaohsiung J Med Sci 2020.

26 Haj-Salem I, Fakhfakh R, Berube JC, et al. MicroRNA-19a enhances proliferation of BEC by targeting TGFbetaR2 gene in severe asthma [J]. Allergy. 2015;70(2):212-9.

27 Shi J, Chen M, Ouyang L, et al. miR-142-5p and miR-130a-3p Regulate Pulmonary macrophage polarization and asthma airway remodeling [J]. Immunol Cell Biol 2020.

28 Dimasuay KG, Schaunaman N, Martin RJ, et al. Parkin, an E3 ubiquitin ligase, enhances airway mitochondrial DNA release and inflammation [J]. Thorax 2020. 